\title{
Wavelet Sampling Theorems for Irregularly Sampled Signals
}

\author{
Wen Chen and Shuichi Itoh \\ Graduate School of Information Systems, University of Electro-Communications, Tokyo, Japan 182
}

Junji Shiki

Department of Electronic Engineering, University of Electro-Communications, Tokyo, Japan 182

\section{SUMMARY}

A formula for recovering the original signal from its irregularly sampled values using wavelets, which extends the Walter sampling theorem to the irregular sampling case and generalizes the Paley-Wiener 1/4-Theorem by removing the symmetricity constraint for sampling, is presented.@ 1999 Scripta Technica, Electron Comm Jpn Pt 3, 82(5): 65-71, 1999

Key words: Wavelet; sampling theorem; scaling function; orthogonality; biorthogonality.

\section{Introduction}

In digital signal and image processing, digital communications, and so forth, a continuous signal is usually represented and processed by using its discrete samples. How, then, are we to reconstruct the original signal from its discrete samples? The classical Shannon sampling theorem gives the following formula for band-limited finite energy signals.

For a finite energy $\sigma$-band continuous signal $f(t), t \in R$, that is, $\operatorname{supp} \hat{f}(\omega) \subset[-\sigma, \sigma]$ and $f \in L^{2}(\mathrm{R})$, it can be recovered by the formula

$$
\begin{gathered}
f(t)=\sum_{n \in \mathrm{Z}} f(n T) \frac{\sin \sigma(t-n T)}{\sigma(t-n T)} \\
T \leq \frac{\pi}{\sigma}, \quad t \in \mathrm{R}
\end{gathered}
$$

where $\hat{f}$ is the Fourier transform of $f(t)$ defined by

$$
\begin{aligned}
\mathcal{F}[f(t)](\omega)= & \hat{f}(\omega)=\int_{\mathrm{R}} f(t) e^{-i \omega t} d t \\
& f \in L^{1}(\mathrm{R}) \cap L^{2}(\mathrm{R})
\end{aligned}
$$

If we let $\sigma=2^{m} \pi, m \in \mathrm{Z}$, Shannon sampling can be viewed as a special case of sampling in wavelet subspaces, with $\varphi(t)=\sin \pi t / \pi t$ playing the role of the scaling function of MRA (multiresolution analysis) = $\left\{V_{m}=\overline{\operatorname{span}}\left\{\varphi\left(2^{m} t-n\right)\right\}_{n}\right\}_{m}$. The dilation equation is

$$
\varphi(t)=\sum_{k} \frac{\sin \pi k / 2}{\pi k / 2} \varphi(2 t-k), \quad t \in \mathrm{R}
$$

Realizing this property, Walter [4] established a sampling theorem for a class of wavelet subspaces. Let $\varphi(t)$ be a continuous scaling function of an MRA $\left\{V_{m}\right\}_{m}$ such that $\varphi(t) \leq O\left(|t|^{-1-\varepsilon}\right)$ for some $\varepsilon>0$ when $|t| \rightarrow \infty, q(s, t)$ $=\Sigma_{n} \varphi(s-n) \varphi(t-n)$, and $\hat{\varphi}^{*}(\omega)=\Sigma_{n} \varphi(n) e^{i n \omega} \neq 0$. Walter showed that, in the orthonormal case, $\{q(s, n)\}_{n \in Z}$ is a Riesz basis of $V_{0}$. Furthermore, let $\left\{S_{n}(t)\right\}_{n \in \mathrm{Z}}$ be biorthogonal to 
$\{q(s, n)\}_{n \in \mathrm{Z}}$. Then $S_{n}(t)=S_{0}(t-n)$ holds and $f \in V_{0}$ can be recovered by the formula

$$
f(t)=\sum_{n \in Z} f(n) S_{0}(t-n)
$$

Following the Walter sampling theorem, to recover the original signals from their discrete samples we can use many proper scaling functions other than the sinc function.

However, in real applications, sampling is not always strictly regular. The sampling interval of natural sampling fluctuates according to the signals sampled. There may be cases where undesirable jitter exists in the sampling instants. Some communication systems may suffer from random delay due to channel traffic congestion or encoding delay. In such cases, system design will be made easier if irregular sampling is tolerated. Thus, irregular sampling may help design signal processing systems, and open the possibilities of new systems that have been considered difficult so far.

Then how are we to deal with these cases in which sampling is not regular but irregular? The famous PaleyWiener 1/4-Theorem (see Young [6]) states that there is a $\left\{S_{k}(t)\right\}_{k} \subset V_{0}$ such that for any $f \in V_{0}$,

$$
f(t)=\sum_{k} f\left(t_{k}\right) S_{k}(t)
$$

if $t_{k}=-t_{-k}$ for $k=0,1,2, \ldots$, and $\left|t_{k}-k\right| \leq l<1 / 4$.

In this paper, we generalize the Paley-Wiener theorem and establish sampling theorems for irregularly sampled signals in orthonormal and biorthonormal wavelet subspaces by removing the symmetricity constraint $t_{k}=-t_{-k}$. Moreover, the result is shown to hold for general wavelet subspaces and an example is also calculated to show the result.

\section{Sampling in Orthonormal Wavelet Subspaces}

First we consider the simplest case of sampling in orthonormal wavelet subspaces and establish a similar formula for it.

Theorem 1 Let $\varphi(t)$ be an orthonormal continuous scaling function of MRA $\left\{V_{m}\right\}_{m}$ such that

1. $\varphi(t) \leq O\left(1 /|t|^{1+\varepsilon}\right)$ for some $\varepsilon>0$ when $|t| \rightarrow \infty$,

2. $\varphi(t)$ is differentiable in each interval $(n, n+1)$, and $\Sigma_{n} \sup _{(n, n+1)}\left|\varphi^{\prime}(t)\right|<\infty$,
3. $\hat{\varphi}^{*}(\omega) \neq 0$.

Then there is a $\left\{S_{n}(t)\right\}_{n} \subset V_{0}$ such that $f(t) \in V_{0}$ can be recovered by the formula

$$
f(t)=\sum_{n} f\left(n+\delta_{n}\right) S_{n}(t)
$$

if sequence $\left\{\delta_{n}\right\}_{n} \subset(-1,1)$ and

$$
\sum_{n}\left|\delta_{n}\right|<\frac{\inf _{0 \leq \omega \leq 2 \pi}\left|\hat{\varphi}^{*}(\omega)\right|}{\sum_{n} \sup _{U_{o}(n, \delta)}\left|\varphi^{\prime}(x)\right|}
$$

where

$$
\delta=\sup _{k}\left|\delta_{k}\right|, \quad \sum_{n} \sup _{U_{o}(n, \delta)}\left|\varphi^{\prime}(x)\right| \neq 0
$$

and $U_{o}(n, \delta)$ is the $\delta$-neighborhood of $n$ except for $n$ itself.

In order to prove Theorem 1 we need two lemmas (Lemma 1 can be found on p. 46 of Ref. 2).

Lemma 1 (see Theorem 9 in Ref. 2) Let $\left\{z_{n}\right\}_{n}$ be a basic sequence ${ }^{1}$ in Banach space $(X,\|\cdot\|)$ and let $\left\{z_{n}^{*}\right\}_{n}$ be the coefficient functional ${ }^{2}$ of $\left\{z_{n}\right\}_{n}$ extended to $X$ in the HahnBanach sense. Then $\left\{y_{n}\right\}_{n}$ is an equivalent basic sequence of $\left\{z_{n}\right\}_{n}$ in $X$ if

$$
\sum_{n}\left\|z_{n}^{*}\right\|\left\|z_{n}-y_{n}\right\|<1
$$

The following are, respectively, two norms in $L^{2}(\mathrm{R})$ and $L^{2}(0,2 \pi)$ :

$$
\|f\|=\left(\int_{\mathrm{R}}|f(t)|^{2} d t\right)^{1 / 2}
$$

and

$$
\|f\|_{L^{2}(0,2 \pi)}=\left(\int_{0}^{2 \pi}|f(t)|^{2} d t\right)
$$

Lemma 2 Let $\varphi(t)$ be an orthonormal continuous scaling function of MRA $\left\{V_{m}\right\}_{m}$ such that $\varphi(t)=O\left(1 /|t|^{1+\varepsilon}\right)$ for some $\varepsilon>0$ when $|t| \rightarrow \infty$. Then for $f(t) \in V_{0}$,

$$
f(t)=\int_{\mathbf{R}} f(s) q(s, t) d s
$$

\footnotetext{
${ }^{1}\left\{z_{n}\right\}_{n}$ is called a basic sequence when it is a basis of the Banach space span $\left\{z_{n}\right\}_{n}$.

${ }^{2}$ Coefficient functional $z_{n}^{*}$ is defined as $x=\Sigma_{n} z_{n}^{*}(x) z_{n}$ for any $x \in$ span $\left\{z_{n}\right\}_{n}$.
} 
Proof The assumption $\varphi(t)=O\left(1 /|t|^{1+\varepsilon}\right)$ implies that $q(s, t)=\Sigma_{n} \varphi(s-n) \varphi(t-n)$ is well-defined. Since $\{\varphi(t-n)\}_{n} \in l^{2}$ and $\{\varphi(s-n)\}_{n}$ is orthonormal, we have

$$
q(t, s)=\sum_{n} \varphi(t-n) \varphi(s-n) \in L^{2}(\mathrm{R}(s))
$$

for a specified $t \in \mathrm{R}$. Thus, $f(s) q(s, t) \in L^{1}(\mathrm{R}(s))$ for any $f(s) \in V_{0} \subset L^{2}(\mathrm{R})$ and a specified $t \in \mathrm{R}$. Moreover, $\varphi(t)=O\left(1 /|t|^{1+\varepsilon}\right)$ also implies that $\varphi(s-n)$ is uniformly bounded and $\Sigma_{n}|\varphi(t-n)|$ is uniformly convergent with respect to a specified $s \in \mathrm{R}$. Therefore,

$$
\sum_{n} \varphi(t-n) \varphi(s-n)
$$

is uniformly convergent with respect to $s \in \mathrm{R}$. From Eqs. (7), (8), and the orthonormality of $\{\varphi(t-n)\}_{n}$, we derive

$$
\begin{array}{rl}
\int_{\mathrm{R}} & f(s) q(t, s) d s \\
& =\int_{\mathrm{R}} f(s) \sum_{n} \varphi(t-n) \varphi(s-n) d s \\
& =\sum_{n} \varphi(t-n) \int_{\mathrm{R}} f(s) \varphi(s-n) d s \\
& =f(t)
\end{array}
$$

Proof of Theorem 1 When $\hat{\varphi}^{*}(\omega) \neq 0$ holds, $\{q(t, k)\}_{k}$ is a Riesz basis of $V_{0}$ (see Walter [4]). Let $\hat{V}_{0}$ be the image space of $V_{0}$ under the mapping Fourier transform defined in Eq. (2). Since the Fourier transform is isometric ${ }^{3}$ modulo a coefficient $\sqrt{2 \pi}$, we can be sure that $\{\hat{q}(\omega, k)\}_{k}$ is a Riesz basis of $V_{0}$.

In order to show that $\left\{q\left(t, t_{k}\right)\right\}_{k}$ with $t_{k}=k+\delta_{k}$ is a Riesz basis of $V_{0}$, it is enough if we can show that $\left\{\hat{q}\left(\omega, t_{k}\right)\right\}_{k}$ is a Riesz basis of $\hat{V}_{0}$. On one hand,

$$
\begin{aligned}
\hat{q}(\omega, k) & =\sum_{n} \varphi(k-n) \mathcal{F}[\varphi(t-n)](\omega) \\
& =\sum_{n} \varphi(k-n) \hat{\varphi}(\omega) e^{-i n \omega} \\
& =\left(\sum_{n} \varphi(n) e^{i n \omega}\right) \hat{\varphi}(\omega) e^{-i k \omega} \\
& =\hat{\varphi}^{*}(\omega) g_{k}(\omega)
\end{aligned}
$$

where $\hat{\varphi}^{*}(\omega)$ and $g_{k}(\omega)$ are defined by

$$
\begin{aligned}
\hat{\varphi}^{*}(\omega) & =\sum_{n} \varphi(n) e^{i n \omega} \\
g_{k}(\omega) & =\hat{\varphi}(\omega) e^{-i k \omega}
\end{aligned}
$$

For the same reason, we have

$$
\hat{q}\left(\omega, t_{k}\right)=\hat{\varphi}_{\delta_{k}}^{*}(\omega) g_{k}(\omega)
$$

where $\hat{\varphi}_{\delta_{k}}^{*}(\omega)$ is defined by

$$
\hat{\varphi}_{\delta_{k}}^{*}(\omega)=\sum_{n} \varphi\left(n+\delta_{k}\right) e^{i n \omega}
$$

From Lemma 1, we need only show

$$
\Delta=\sum_{k}\left\|q_{k}^{*}\right\|\left\|\hat{q}\left(\omega, t_{k}\right)-\hat{q}(\omega, k)\right\|<1
$$

where $\left\{q_{k}^{*}\right\}_{k}$ is the coefficient functional of $\{\hat{q}(\omega, k)\}_{k}$. But for any $\left\{c_{k}\right\}_{k} \in l^{2}$,

$$
\begin{aligned}
& \left\|\sum_{k} c_{k} \hat{q}(\omega, k)\right\|=\left\|\hat{\varphi}^{*}(\omega) \sum_{k} c_{k} \hat{\varphi}(\omega) e^{-i k \omega}\right\| \\
& \quad \geq \inf \left|\hat{\varphi}^{*}(\omega)\right|\left\|\mathcal{F}\left[\sum_{k} c_{k} \varphi(t-k)\right](\omega)\right\| \\
& \quad=\sqrt{2 \pi} \inf \left|\hat{\varphi}^{*}(\omega)\right|\left(\sum_{k}\left|c_{k}\right|^{2}\right)^{1 / 2} \\
& \quad \geq \sqrt{2 \pi} \inf \left|\hat{\varphi}^{*}(\omega)\right|\left|c_{k_{0}}\right| \\
& =\sqrt{2 \pi} \inf \left|\hat{\varphi}^{*}(\omega)\right|\left|q_{k_{0}}^{*}\left(\sum_{k} c_{k} \hat{q}(\omega, k)\right)\right|
\end{aligned}
$$

where Eq. (17) is due to the orthonormality of $\varphi(t)$ and the Parseval identity. Therefore,

$$
\left\|q_{k_{0}}^{*}\right\| \leq\left(\sqrt{2 \pi} \inf \left|\hat{\varphi}^{*}(\omega)\right|\right)^{-1}
$$

From Eqs. (11), (14), and (18),

$$
\begin{aligned}
\Delta & \leq \frac{\sum_{k}\left\|\left(\hat{\varphi}_{\delta_{k}}^{*}(\omega)-\hat{\varphi}^{*}(\omega)\right) g_{k}(\omega)\right\|}{\sqrt{2 \pi} \inf \left|\hat{\varphi}^{*}(\omega)\right|} \\
& =\frac{\sum_{k}\left\|\sum_{n}\left(\varphi\left(n+\delta_{k}\right)-\varphi(n)\right) e^{i n \omega} \hat{\varphi}(\omega) e^{-i k \omega}\right\|}{\sqrt{2 \pi} \inf \left|\hat{\varphi}^{*}(\omega)\right|} \\
& \leq \frac{\sum_{k} \sum_{n}\left|\varphi\left(n+\delta_{k}\right)-\varphi(n)\right|\|\hat{\varphi}\|}{\sqrt{2 \pi} \inf \left|\hat{\varphi}^{*}(\omega)\right|}
\end{aligned}
$$

On the other hand, since $\varphi$ is differentiable in each interval $(n, n+1)$ and orthonormal, the Lagrange mean formula implies

$$
\begin{aligned}
\sum_{k} \sum_{n} \mid \varphi(n & \left.+\delta_{k}\right)-\varphi(n) \mid \\
& =\sum_{k}\left|\delta_{k}\right| \sum_{n}\left|\varphi^{\prime}\left(\xi_{n, k}\right)\right|
\end{aligned}
$$


where $\xi_{n, k}$ is the Lagrange mean point in $\left(n, n+\delta_{k}\right)$. By virtue of $\|\hat{\varphi}\|=\sqrt{2 \pi}\|\varphi\|=\sqrt{2 \pi}$, Eqs. (16), (19), and (20), in order to show that $\left\{q\left(t, t_{k}\right)\right\}_{k}$ is a Riesz basis of $V_{0}$, we need only show

$$
\frac{\sqrt{2 \pi} \sum_{k}\left|\delta_{k}\right| \sum_{n} \sup _{U_{o}(n, \delta)}\left|\varphi^{\prime}(x)\right|}{\sqrt{2 \pi} \inf \left|\hat{\varphi}^{*}(\omega)\right|}<1
$$

that is,

$$
\sum_{k}\left|\delta_{k}\right|<\frac{\inf \left|\hat{\varphi}^{*}(\omega)\right|}{\sum_{n} \sup _{U_{o}(n, \delta)}\left|\varphi^{\prime}(x)\right|}
$$

This is exactly the given Eq. (6). Let $\left\{S_{k}(t)\right\}_{k}$ be biorthonormal to $\left\{q\left(t, t_{k}\right\}_{k}\right.$ in $V_{0}$. Then Lemma 2 implies that for any $f(t) \in V_{0}$,

$$
f(t)=\sum_{k}\left\langle f(t), q\left(t, t_{k}\right)\right\rangle S_{k}(t)=\sum_{k} f\left(t_{k}\right) S_{k}(t)
$$

\section{Remark 1}

[5]), $\hat{\varphi}^{*}(\omega)=1$ holds.

1. When $\varphi$ is a cardinal scaling function (see Walter

2. Obviously the condition $\Sigma_{n} \sup _{(n, n+1)}\left|\varphi^{\prime}(t)\right|<\infty$ can be replaced by $\left.\varphi^{\prime}(t)\right|_{(n, n+1)}=O\left(1 /|n|^{1+\varepsilon}\right)$ for some $\varepsilon>0$ when $|t| \rightarrow \infty$. But $\varphi^{\prime}(t)=O\left(1 /|t|^{1+\varepsilon}\right)$ is easier to verify.

3. In practical cases, we can obtain only finite samples. For example, $t \in[-N, N]$. The Paley-Wiener [6] theorem requires that $\delta_{k}=-\delta_{-k}$ and $|\delta k|<1 / 4$. But we only require that Eq. (6) hold instead of the restrictive constraint imposed on each $\delta_{k},(k \in[-N, N] \cap \mathrm{Z})$, where the bound of Eq. (6) depends on $\delta=\sup _{k}\left|\delta_{k}\right|$.

4. When $f$ is not in $V_{0}$ we also can use the formula to recover it, but the aliasing error must be estimated (see Walter [5]).

5. $\left\{S_{k}(t)\right\}_{k}$ can be obtained by calculating the biorthogonal basis of $\left\{q\left(t, k+\delta_{k}\right)\right\}_{k}$. For regular sampling, $\delta_{k}=0$. Hence, Eq. (5) is the same as Eq. (4).

\section{Sampling in Biorthogonal Wavelet Subspaces}

When $\varphi(t)$ is not orthonormal but biorthogonal ${ }^{4}$, Theorem 1 cannot hold. But we can find a similar formula for it.

Theorem 2 Let $\{\varphi(t), \widetilde{\varphi}(t)\}$ be the continuous scaling function pair for biorthogonal MRA $\left\{V_{m}, \widetilde{V}_{m}\right\}$ (with $V_{0}=\widetilde{V}_{0}$ ) such that

\footnotetext{
${ }^{4}$ See Cohen and colleagues [1] or Long and Chen [3] for biorthogonal
} wavelets.
1. $\varphi(t)=O\left(1 /|t|^{1+\varepsilon}\right), \tilde{\varphi}(t)=O\left(1 /|t|^{1+\varepsilon}\right)$ for some $\varepsilon>$ 0 when $|t| \rightarrow \infty$,

2. $\tilde{\varphi}(t)$ is differentiable in each interval $(n, n+1)$, and $\sum_{n} \sup _{(n, n+1)}\left|\tilde{\varphi}^{\prime}(x)\right|<\infty$,

3. $\hat{\widetilde{\varphi}}^{*}(\omega) \neq 0$.

Then there is a $\left\{S_{n}(t)\right\}_{n} \subset V_{0}$ such that for any $f(t) \in V_{0}$,

$$
f(t)=\sum_{n} f\left(n+\delta_{n}\right) S_{n}(t)
$$

if $\delta_{n} \in(-1,1)$ and

$$
\sum_{n}\left|\delta_{n}\right|<\frac{\sqrt{2 \pi} \inf _{\omega}\left|\hat{\tilde{\varphi}}^{*}(\omega) G(\varphi)(\omega)\right|}{\|G(\varphi)\|_{L^{2}(0,2 \pi)} \sum_{n} \sup _{U_{o}(n, \delta)}\left|\tilde{\varphi}^{\prime}(x)\right|}
$$

where

$$
\delta=\sup _{k}\left|\delta_{k}\right|, \quad \sum_{n} \sup _{U_{o}(n, \delta)}\left|\tilde{\varphi}^{\prime}(x)\right| \neq 0
$$

$G(\varphi)=\left(\Sigma_{k}|\hat{\varphi}(\omega+2 k \pi)|^{2}\right)^{1 / 2}$, and $\hat{\tilde{\varphi}}^{*}(\omega)=\Sigma_{n} \widetilde{\varphi}(n) e^{i n \omega}$.

Proof Let

$$
q(t, s)=\sum_{n} \varphi(t-n) \tilde{\varphi}(s-n)
$$

For the same reason as for Lemma 2, we know $f(t)=$ $\int_{\mathrm{R}} f(s) q(t, s) d s$ for $f(t) \in V_{0}$ and that $g(s)=\int_{\mathrm{R}} g(t) q(t, s) d t$ for $g(s) \in \widetilde{V}_{0}$. When $\hat{\varphi}^{*}(\omega) \neq 0$, it is easy to see, by referring to Walter [4], that $\{q(t, k)\}_{k}$ is a Riesz basis of $V_{0}$, or equivalently $\{\hat{q}(\omega, k)\}_{k}$ is a Riesz basis of $V_{0}$. In order to show that $\left\{q\left(t, k+\delta_{k}\right)\right\}_{k}$ is a Riesz basis of $V_{0}$, it is enough to show that $\left\{\hat{q}\left(\omega, k+\delta_{k}\right)\right\}_{k}$ is a Riesz basis of $\hat{V}_{0}$ due to the isometricity of the Fourier transform. Referring to the proof of Theorem 1, we have

$$
\begin{aligned}
\hat{q}(\omega, k) & =\hat{\tilde{\varphi}}^{*}(\omega) g_{k}(\omega) \\
\hat{q}\left(\omega, k+\delta_{k}\right) & =\tilde{\tilde{\varphi}}_{\delta_{k}}^{*}(\omega) g_{k}(\omega)
\end{aligned}
$$

and

$$
\begin{gathered}
\Delta \leq \sum_{k}\left\|q_{k}^{*}\right\|\left\|\hat{q}\left(\omega, k+\delta_{k}\right)-\hat{q}(\omega, k)\right\| \\
\leq \sup _{k}\left\|q_{k}^{*}\right\| \sum_{k} \| \sum_{n}\left(\tilde{\varphi}\left(n+\delta_{k}\right)\right. \\
\leq \sup _{k}\left\|q_{k}^{*}\right\|\|G(\varphi)\|_{L^{2}(0,2 \pi)} \\
\quad \times \sum_{k}\left|\delta_{k}\right| \sum_{n}\left|\tilde{\varphi}^{\prime}\left(\xi_{n, k}\right)\right|
\end{gathered}
$$


where $\xi_{n, k}$ is the Lagrange mean point in $\left(n, n+\delta_{k}\right)$ and $\left\{q_{k}^{*}\right\}_{k}$ is the coefficient functional of $\{\hat{q}(\omega, k)\}_{k}$. But for any $\left\{c_{k}\right\}_{k} \in l^{2}$,

$$
\begin{aligned}
\| \sum_{k} & c_{k} \hat{q}(\omega, k) \| \\
& =\left\|\sum_{k} c_{k} \hat{\tilde{\varphi}}^{*}(\omega) \hat{\varphi}(\omega) e^{-i k \omega}\right\| \\
& =\left\|\hat{\tilde{\varphi}}^{*}(\omega) G(\varphi) \sum_{k} c_{k} e^{-i k \omega}\right\|_{L^{2}(0,2 \pi)} \\
& \geq \sqrt{2 \pi} \inf \left|\hat{\tilde{\varphi}}^{*}(\omega) G(\varphi)\right|\left(\sum_{k}\left|c_{k}\right|^{2}\right)^{1 / 2} \\
& \geq \sqrt{2 \pi} \inf \left|\hat{\tilde{\varphi}}^{*}(\omega) G(\varphi)\right|\left|c_{k_{0}}\right| \\
& =\sqrt{2 \pi} \inf \left|\hat{\tilde{\varphi}}^{*}(\omega) G(\varphi)\right|\left|q_{k_{0}}^{*}\left(\sum_{k} c_{k} \hat{q}(\omega, k)\right)\right|
\end{aligned}
$$

Hence,

$$
\sup _{k}\left\|q_{k}^{*}\right\| \leq\left(\sqrt{2 \pi} \inf \left|\hat{\tilde{\varphi}}^{*} G(\varphi)\right|\right)^{-1}
$$

Let

$$
\begin{aligned}
\Delta & \leq \frac{\|G(\varphi)\|_{L^{2}(0,2 \pi)}}{\sqrt{2 \pi} \inf \left|\tilde{\tilde{\varphi}}^{*} G(\varphi)\right|} \sum_{k}\left|\delta_{k}\right| \sum_{n} \sup _{U_{o}(n, \delta)}\left|\tilde{\varphi}^{\prime}(x)\right| \\
& <1
\end{aligned}
$$

that is,

$$
\sum_{k}\left|\delta_{k}\right|<\frac{\sqrt{2 \pi} \inf \left|\hat{\tilde{\varphi}}^{*} G(\varphi)\right|}{\|G(\varphi)\|_{L^{2}(0,2 \pi)} \sum_{n} \sup _{U_{o}(n, \delta)}\left|\tilde{\varphi}^{\prime}(x)\right|}
$$

Then $\left\{q\left(t, k+\delta_{k}\right)\right\}_{k}$ is a Riesz basis of $V_{0}$. If we take $\left\{S_{k}(t)\right\}_{k}$ as biorthogonal to $\left\{q\left(t, k+\delta_{k}\right)\right\}_{k}$ in $V_{0}$, then for any $f \in V_{0}, \quad$ we have $f(t)=\Sigma_{k}\left\langle f(s), q\left(s, k+\delta_{k}\right)\right\rangle S_{k}(t)$ $=\Sigma_{k} f\left(k+\delta_{k}\right) S_{k}(t)$.

\section{Remark 2}

1. When $\varphi(t)$ is orthonormal, $G(\varphi)=1$ holds. Thus, Eq. (28) is the same as Eq. (22).

2. Since $\varphi$ and $\tilde{\varphi}$ are symmetric, Theorem 2 still holds when $\varphi$ and $\widetilde{\varphi}$ change their positions.

3. Due to $\|G(\varphi)\|_{L^{2}(0,2 \pi)} \leq \sqrt{2 \pi} \sup \mid G(\varphi)$ l, Eq. (28) can be replaced by

$$
\sum_{k}\left|\delta_{k}\right|<\frac{\inf \left|\hat{\tilde{\varphi}}^{*} G(\varphi)\right|}{\sup |G(\varphi)| \sum_{n} \sup _{U_{o}(n, \delta)}\left|\tilde{\varphi}^{\prime}(x)\right|}
$$

In many practical cases, the scaling function is neither orthonormal nor biorthogonal. But we need to use these scaling functions in recovering signals; the following corollary is designed for these cases.

Corollary 1 With the same assumption as Theorem 1 except that $\varphi(t)$ is not orthonormal, for any $f(t) \in V_{0}$, there is an $S_{k}(t) \in V_{0}$ such that

$$
f(t)=\sum_{k} f\left(k+\delta_{k}\right) S_{k}(t)
$$

if $\delta_{k} \in(-1,1)$ and

$$
\begin{aligned}
\sum_{k}\left|\delta_{k}\right|<\frac{\sqrt{2 \pi} \inf \left|\hat{\varphi}^{*}(\omega) / G(\varphi)\right|}{\left\|\left(\hat{\varphi}^{*}(-\omega) G(\varphi)\right)^{-1}\right\|_{L^{2}(0,2 \pi)}} \\
\quad \times \frac{1}{\sum_{n} \sup _{U_{o}(n, \delta)}\left|\sum_{k} \varphi^{\prime}(x-k) \varphi(-k)\right|}
\end{aligned}
$$

where $\sum_{n} \sup _{U_{o}(n, \delta)}\left|\Sigma_{k} \varphi^{\prime}(x-k) \varphi(-k)\right| \neq 0$, and $G(\varphi)=$ $\left(\Sigma_{k}|\hat{\varphi}(\omega+2 k \pi)|^{2}\right)^{1 / 2}$.

Proof Let $q(s, t)=\Sigma_{n} \varphi(s-n) \varphi(t-n)$. When $\hat{\varphi}^{*}(\omega) \neq 0$ holds, $\{q(s, k)\}_{k}$ is a Riesz basis of $V_{0}$. Suppose that $\left\{\widetilde{q}_{k}(s)\right\}_{k}$ is biorthogonal to $\{q(t, k)\}_{k}$ in $V_{0}$. Walter [5] states that $\widetilde{q}_{k}(s)=\widetilde{q}_{0}(s-k)$ and $q(s, k)=q(s-k, 0)$. Thus $\left\{q(s, 0), \widetilde{q}_{0}(s)\right\}$ is a biorthogonal scaling function pair for $\left\{V_{0}, V_{0}\right\}$. Referring to Theorem 2 and point 2 of Remark 2, there is a $\left\{S_{k}(t)\right\}_{k} \in V_{0}$ such that for any $f \in V_{0}$,

$$
f(t)=\sum_{k} f\left(k+\delta_{k}\right) S_{k}(t)
$$

if

$$
\sum_{k}\left|\delta_{k}\right|<\frac{\sqrt{2 \pi} \inf \left|\hat{q}^{*}(\cdot, 0) G\left(\tilde{q}_{0}\right)\right|}{\left\|G\left(\tilde{q}_{0}\right)\right\|_{L^{2}(0,2 \pi)} \sum_{n} \sup _{U_{o}(n, \delta)}\left|q^{\prime}(x, 0)\right|}
$$

where

$$
\begin{aligned}
\hat{q}^{*}(\omega, 0) & =\sum_{m} \sum_{n} \varphi(m-n) \varphi(-n) e^{i m \omega} \\
& =\hat{\varphi}^{*}(\omega) \hat{\varphi}^{*}(-\omega) \\
q^{\prime}(x, 0) & =\sum_{n} \varphi^{\prime}(x-n) \varphi(-n)
\end{aligned}
$$

But from Theorem 9.2 of Walter [5],

$$
\hat{\tilde{q}}_{0}(\omega)=\hat{\varphi}(\omega) /\left(\hat{\varphi}^{*}(-\omega) G^{2}(\varphi)\right)
$$


On the other hand, we also have

$$
\begin{aligned}
G\left(\tilde{q}_{0}\right)^{2} & =\sum_{n}\left|\hat{\tilde{q}}_{0}(\omega+2 n \pi)\right|^{2} \\
& =\sum_{n}\left|\frac{\hat{\varphi}(\omega+2 n \pi)}{\hat{\varphi}^{*}(-\omega) G^{2}(\varphi)}\right|^{2} \\
& =\frac{\sum_{n}|\hat{\varphi}(\omega+2 n \pi)|^{2}}{\left|\hat{\varphi}^{*}(-\omega)\right|^{2} G^{4}(\varphi)} \\
& =\frac{1}{\left|\varphi^{*}(-\omega)\right|^{2} G^{2}(\varphi)}
\end{aligned}
$$

From Eqs. (30), (31), and (32), Eq. (29) is equivalent to

$$
\begin{aligned}
\sum_{k}\left|\delta_{k}\right|< & \frac{\sqrt{2 \pi} \inf \left|\hat{\varphi}^{*}(\omega) / G(\varphi)\right|}{\left\|\left(\hat{\varphi}^{*}(-\omega) G(\varphi)\right)^{-1}\right\|_{L^{2}(0,2 \pi)}} \\
& \times \frac{1}{\sum_{n} \sup _{U_{o}(n, \delta)}\left|\sum_{k} \varphi^{\prime}(x-k) \varphi(-k)\right|}
\end{aligned}
$$

\section{Remark 3}

1. In practical cases we can select the proper scaling function $\varphi(t)$ such that the right side of Eq. (33) is big enough to cover the necessary fluctuation $\operatorname{range} \sup _{k}\left\{\delta_{k}\right\}$.

2. But the theorem is still useful even if the right side of Eq. (33) is not so big. In many practical cases of sampling the irregular deviation in the sampling points is only slight. As long as Eq. (33) is satisfied, the original signal can be reconstructed. However, the Paley-Wiener [6] method cannot be applied, since it needs constraint $t_{k}=-t_{-k}$.

3. For example, take the B-spline $\varphi(t)=N_{2}(t)=t \mathbf{1}_{[0,1)}(t)+(2-t) \mathbf{1}_{[1,2)}(t)$. Then the right side of (33) becomes $\sqrt{2 \pi} /\left(3\left\|G^{-1}(\varphi)\right\|_{L^{2}(0,2 \pi)}\right)$ where $G(\varphi)=\sqrt{\frac{1}{3}+\frac{2}{3} \cos ^{2} \omega / 2}$. Due to $\left\|G^{-1}(\varphi)\right\|_{L^{2}(0,2 \pi)} \leq \sqrt{6 \pi}$, we derive $\sqrt{2 \pi} /\left(3\left\|G^{-1}(\varphi)\right\|_{L^{2}(0,2 \pi)}\right) \geq 1 / 3 \sqrt{3}$. Then the irregularly sampled signals in $V_{0}$ can be recovered if $\Sigma_{k}\left|\delta_{k}\right|<1 / 3 \sqrt{3}$. But the Paley-Wiener constraint $t_{k}=-t_{-k}$ for sampling is not necessary here.

4. In order for the right side of Eq. (33) to be big enough, the scaling function $\varphi(t)$ should be selected such that $\left|\varphi^{\prime}(x)\right|$ is small enough. But a small $\left|\varphi^{\prime}(x)\right|$ implies that we can only reconstruct the low-frequency signals. Therefore, selecting a proper scaling function, such that the permitted deviation $\delta_{k}$ and wavelet subspaces can be big enough, is an important factor for signal reconstruction in wavelet subspaces by the theorem.

\section{Conclusions}

In this paper, a reconstruction formula for irregularly sampled signals in general wavelet subspaces is established. Compared to the Paley-Wiener theorem, the symmetricity constraint is removed.

The B-spline of order 2 is calculated as an example to demonstrate the theorem.

It is interesting to know what class of scaling functions can allow a large margin of sampling irregularity. The important case is that when the class of wavelet subspaces is rich enough to include many interesting functions in application.

Acknowledgments. The authors thank Professor T. Hashimoto, Dr. H. Tsuchiya, and the anonymous referees for their helpful suggestions.

\section{REFERENCES}

1. Cohen A, Daubechies I, Feauveau JC. Biorthogonal bases of compactly supported wavelets. Commun Pure Appl Math 1992;45:485-560.

2. Diestel J. Sequences and series in Banach spaces. Springer-Verlag; 1984.

3. Long R, Chen D. Biorthogonal wavelet bases on $R^{d}$. Appl Comp Harmonic Anal 1995;2:230-242.

4. Walter GG. A sampling theorem for wavelet subspaces. IEEE Trans Inf Theory 1992;38:881-884.

5. Walter GG. Wavelets and other orthogonal systems with applications. CRC Press; 1994.

6. Young R. An introduction to non-harmonic Fourier series. Academic Press; 1980. 
AUTHORS (from left to right)
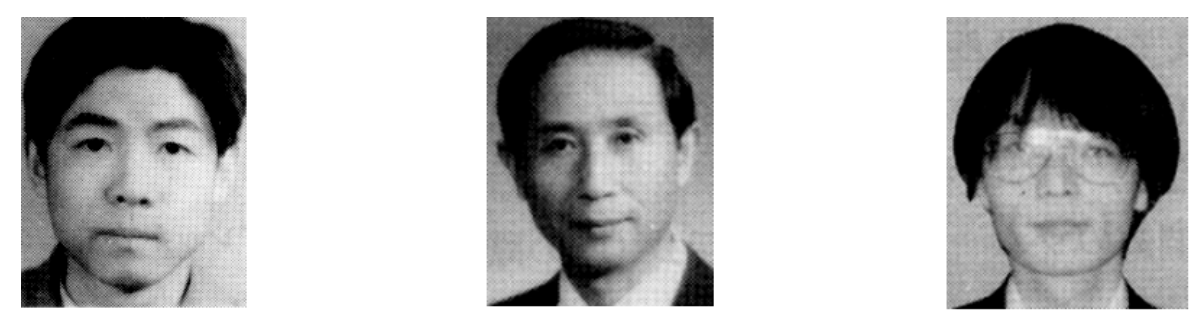

Wen Chen received his B.S. and M.S. degrees in mathematical sciences from Wuhan University, Wuhan, China, in 1990 and 1993, respectively. He joined the Institute of Mathematics, Academia Sinica, Beijing, China, in 1993 and is now studying for his D.Eng. degree at the Graduate School of Information Systems, University of Electro-Communications, Tokyo. His research interests include information theory, wavelet theory, and their applications.

Shuichi Itoh (member) received his B.E., M.E., and D.Eng. degrees in electrical engineering from the University of Tokyo in 1964, 1966, and 1969, respectively. He is now a professor at the Graduate School of Information Systems, University of Electro-Communications. His research interests include data compression, pattern classification, and information theory. He is a member of IEEE, IPSJ, and the Society of Information Theory and Its Applications.

Junji Shiki received his B.E. and M.E. degrees in electronic communications from the University of Electro-Communications in 1992 and 1994, respectively. He is now studying for his D.Eng. degree. His research interests include information theory and its applications. 